\title{
Area Green Efficiency (AGE) of Two Tier Heterogeneous Cellular Networks
}

\author{
Hina Tabassum*, Muhammad Zeeshan Shakir ${ }^{\dagger}$, and Mohamed-Slim Alouini* \\ *Computer, Electrical, Mathematical Sciences and Engineering (CEMSE) Division, \\ King Abdullah University of Science and Technology (KAUST), Thuwal, Makkah Province, Saudi Arabia. \\ ${ }^{\dagger}$ Department of Electrical and Computer Engineering, Texas A\&M University at Qatar (TAMUQ), Doha, Qatar. \\ \{hina.tabassum, slim.alouini\}@kaust.edu.sa, muhammad.shakir@qatar.tamu.edu
}

\begin{abstract}
Small cell networks are becoming standard part of the future heterogeneous networks. In this paper, we consider a two tier heterogeneous network which promises energy savings by integrating the femto and macro cellular networks and thereby reducing $\mathrm{CO}_{2}$ emissions, operational and capital expenditures (OPEX and CAPEX) whilst enhancing the area spectral efficiency (ASE) of the network. In this context, we define a performance metric which characterize the aggregate energy savings per unit macrocell area and is referred to as area green efficiency (AGE) of the two tier heterogeneous network where the femto base stations are arranged around the edge of the reference macrocell such that the configuration is referred to as femto-on-edge (FOE). The mobile users in macro and femto cellular networks are transmitting with the adaptive power while maintaining the desired link quality such that the energy aware FOE configuration mandates to (i) save energy, and (ii) reduce the co-channel interference. We present a mathematical analysis to incorporate the uplink power control mechanism adopted by the mobile users and calibrate the uplink ASE and AGE of the energy aware FOE configuration. Next, we derive analytical expressions to compute the bounds on the uplink ASE of energy aware FOE configuration and demonstrate that the derived bounds are useful in evaluating the ASE under worst and best case interference scenarios. Simulation results are produced to demonstrate the ASE and AGE improvements in comparison to macro-only and macro-femto configuration with uniformly distributed femtocells.
\end{abstract}

Index Terms-Heterogenous networks, area green efficiency, area spectral efficiency, femtocell networks, power control.

\section{INTRODUCTION}

Heterogeneous networks emerges as a striking solution to the challenging demands such as high data-rate transmission, high spectral and power efficiency, improved cell coverage, and cell edge performance of the future wireless networks. Heterogeneous network unites and support diverse entities such as multiple radio access technologies (RATs), architectures, and allows flexible setup of user deployed base stations (BSs) such as micros, femtos, and picos to enhance the network coverage and capacity [1]-[3]. A key recent trend in this regard is the use of femtocells overlaid throughout the traditional macro cellular network which facilitates the operation and transmission of the macrocell BSs in various perspectives. Firstly, the femtocell deployment shares the traffic load of macrocells whilst alleviating the capital and operational expenditures (CAPEX and OPEX) of macrocell BSs [1], [4]. Moreover, the mobile users located within a given femtocell enjoys high quality transmission due to their short distance from the serving BS, hence ensuring enhanced network capacity. The femtocell technology has also been praised as greener, i.e., more energy efficient technology due to the short distance communication [5]-[7].
Several macro-femto deployment strategies are currently under consideration and their performance is commonly calibrated with respect to the achievable profitability, spectral efficiency, and outage probability [7]. However, energy efficiency has been recently marked as one of the alarming bottleneck in the telecommunication growth paradigm mainly due to two major reasons i) dramatically varying global climate [8], and ii) slowly progressing battery technology [9], [10]. Therefore, it is of immense importance for the network designers to critically calibrate the energy efficiency of various deployment strategies along with the other performance metrics.

Recently, in [11] we propose two-tier macro-femto deployment in which the femto BSs are arranged around the edge of the reference macrocell. The deployment is referred to as Femto-on-edge (FOE) and has been shown to produce significant area spectral efficiency (ASE) gains compared to macroonly network. In this paper our contribution is three fold; (i) we extend the framework presented in [11] to compute the uplink spectral and energy efficiency of the energy aware FOE configuration by considering that each mobile user is capable of adapting its transmit power autonomously through selfcalibration while maintaining a certain signal power received at the BS [12]. In this context, we define a performance metric which characterize the network power savings per unit macrocell area. We refer this performance metric to as area green efficiency (AGE) of the two tier heterogeneous network, (ii) we demonstrate that the energy aware FOE configuration offers significant ASE and AGE gains in comparison with the macro-only and macro-femto network deployments with uniformly distributed femtocells (UDF), (iii) Taking into account the worst and best case interference scenarios, we derive analytical expressions to compute the bounds on the ASE of energy aware FOE configuration.

The rest of the paper is organized as follows. Section II discusses the system model. In Section III, we calculate the ASE and AGE of the energy aware FOE configuration. Also, bounds on the ASE of energy aware FOE configuration are derived. Section IV presents numerical and simulation results followed by the concluding remarks in Section V.

\section{System Model}

In this section, we explain the network layout, the channel propagation model, the bandwidth partition and the channel allocation strategy of the energy aware FOE configuration.

\section{A. Network Layout}

We consider two tier energy aware heterogeneous network in the uplink as illustrated in Fig. 1 where the integration 
of macro and femto cellular networks has been shown. The first tier of the considered heterogeneous network comprises of circular macrocells each of radius $R_{m}$ [m] with a BS $B_{m}$ deployed at the center and equipped with an omni-directional antenna. Each macrocell is assumed to have $H$ mobile users uniformly distributed over the region bounded by $R_{0}$ and $R_{m}$, where $R_{0}$ denotes the minimum distance between the macrocell mobile user and its serving BS.

The second tier of the heterogeneous network comprises of $N$ circular femtocells each of radius $R_{n}$ [m] with lowpower low-cost user deployed femto BSs $B_{n}$ located at the center. We consider that the femtocells are distributed around the edge of the reference macrocell such that the resultant femtocell deployment is referred to as Femto-on-edge (FOE) configuration. For practical reasons, we calculate the number of femtocells per macrocell as follows

$$
N=\left\{\begin{array}{cl}
\mu \frac{\left(R_{2}^{2}-R_{1}^{2}\right)}{R_{n}^{2}}=\mu \frac{4 R_{m}}{R_{n}} & R_{m}>R_{n} \\
0 & R_{m} \leq R_{n}
\end{array}\right.
$$

where $R_{1}=R_{m}-R_{n}, R_{2}=R_{m}+R_{n}$ and the factor $0<$ $\mu \leq 1$ is referred to as the femtocell population factor (FPF) which control the number of femtocells per macrocell, i.e.,

$$
\mu=\left\{\begin{array}{cc}
0 & \text { off-load femtocells } \\
1 & \text { maximum number of femtocells per macrocell }
\end{array}\right.
$$

The number of mobile users in each femtocell can be given as $F=(H-L) / N$ where $L=\left(H\left(R_{1}^{2}-R_{0}^{2}\right)\right) / R_{m}^{2}$. To be precise, in FOE configuration, $L$ out of $H$ mobile users are uniformly distributed over the region bounded by $R_{0}$ and $R_{1}$ whereas the remaining mobile users, i.e., $H-L$ are reserved for $N$ femtocells. The bandwidth allocated to a macrocell is reused throughout the macrocell network at a distance $D^{\prime}=$ $R_{u}\left(R_{m}+R_{n}\right)$ [m], where $R_{u}$ represents the network traffic load and $R_{u}=2$ for a fully loaded cellular network. The total bandwidth allocated to the femtocell tier is reused in each of the $N$ femtocells within a macrocell.

\section{B. Bandwidth Partition and Channel Allocation}

We consider the spectrum partition based on the proportion of the number of mobile users in the macrocell and the femtocells. Let $w_{t}[\mathrm{~Hz}]$ is the total bandwidth of the available spectrum per cell then the total bandwidth may be divided as

$$
w_{t}=w_{m}+w_{n}
$$

where $w_{m}=w_{t}(L / H)[\mathrm{Hz}]$ and $w_{n}=w_{t}(N F / H)[\mathrm{Hz}]$ are the amount of the spectrum dedicated to the macrocell and the femtocells respectively based on the proportion of the number of active mobile users. The allocated bandwidth of each mobile user in the macro and the femto cellular networks may be respectively calculated as $w_{l, m}=w_{m} / N_{m}$ and $w_{f, n}=$ $w_{n} / N_{n}$; where $N_{m}$ and $N_{n}$ are the number of active serviced channels available per macrocell and femtocell respectively. Moreover, for simplicity we consider that each channel can be allocated to one mobile user at a time and there will not be any mobile user which cannot be serviced by the respective macro or femto BS such that $N_{n}=F$ and $N_{m}=L$.

\section{Energy Aware Channel Propagation Model}

Wireless channel is usually modeled by (i) distance dependent path-loss, (ii) shadowing, and (iii) multipath fading. In
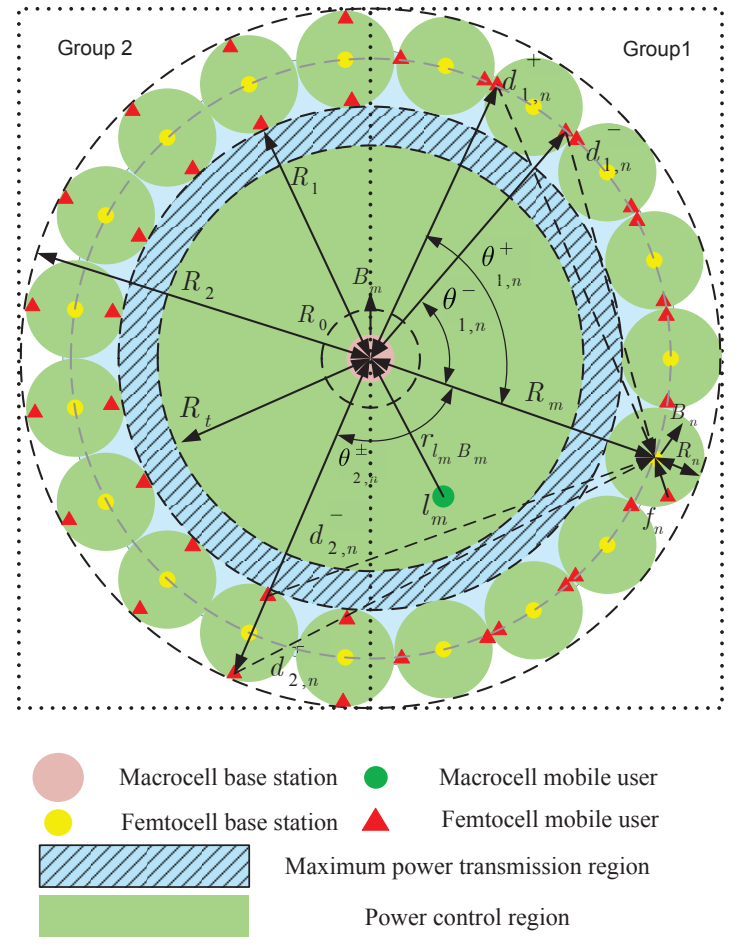

Fig. 1. Graphical illustration of two tier heterogeneous network where a macrocell is surrounded by $N$ femtocells around the edge. The worst and best distances of $N-1$ femtocell interferers are also shown.

this paper, we only consider path-loss effect since we assume a scenario where an efficient antenna diversity combining system is employed at the BS to eliminate the effects of multipath fading [13]. However, this is not a limitation of this work and extensions are possible for shadowing and fading scenarios. We consider two slope path-loss model to obtain the mean received power as a function of the distance between the mobile user and the respective serving BS. In general, the path-loss model can be written as follows

$$
P^{\mathrm{rx}}(r)=\frac{K}{r^{\alpha}(1+r / g)^{\beta}} P^{\mathrm{tx}}
$$

where $P^{r x}[\mathrm{~W}]$ denotes the average received signal power at the reference BS from the desired mobile user which is located at a distance $r$ from the same reference $\mathrm{BS} ; K$ is the constant which is depending on path-loss factor; $P^{\mathrm{tx}}=P_{\max }[\mathrm{W}]$ is the maximum transmit power of each of the mobile user; $\alpha$ is the basic path-loss exponent; $\beta$ is the additional pathloss exponent; and $g=\frac{4 h_{\mathrm{tx}} h_{\mathrm{rx}}}{\lambda_{c}}[\mathrm{~m}]$ is the breakpoint of a path-loss curve and it strictly depends on the BS antenna height $h_{\mathrm{rx}}[\mathrm{m}]$ and the antenna height of mobile user $h_{\mathrm{tx}}[\mathrm{m}]$ and $\lambda_{c}$ is the wave length of the carrier frequency. As each mobile user is considered to be capable of adapting its transmit power autonomously while maintaining a certain signal power received at the BS, the adaptive transmit power can be written as

$$
P^{\mathrm{tx}}=\min \left(P_{\max }, P_{0} \frac{r^{\alpha}(1+r / g)^{\beta}}{K}\right)
$$

where $P_{0}[\mathrm{~W}]$ denotes the signal power received at the BS. Note that (4) is a simplified version of the conventional uplink power control (PC) which is recently approved by 3 GPP in Long Term Evolution (LTE) networks.

The distance at which mobile users require their maximum 
power $P_{\max }$ to fully compensate path-loss while maintaining a certain signal power received at the BS $P_{0}$ is referred to as the threshold distance $\left(R_{t}\right)$. Since the mobile users located within $R_{t}$ can compensate their path-loss while saving some proportion of their power, therefore the region within $R_{t}$ is referred to as green area. However, the mobile users located beyond $R_{t}$ may transmit with their maximum power to achieve some throughput gains. $R_{t}$ can be computed by solving the following equation numerically.

$$
R_{t}^{\alpha}\left(g+R_{t}\right)^{\beta}=\frac{K P_{\max }}{P_{0}} g^{\beta}
$$

For special cases, in which $\beta=\alpha, R_{t}$ can be given as follows

$$
R_{t}=\frac{1}{2}\left(-g \pm \sqrt{g} \sqrt{4\left(\frac{P_{\max } K}{P_{0}}\right)^{1 / \alpha}+g}\right)
$$

Moreover, in the scenarios where $\beta=m \alpha$ and $m$ is an integer, $R_{t}$ can be determined using standard mathematical software packages such as MATHEMATICA.

\section{Mobile User Distribution}

All mobile users are considered mutually independent and uniformly distributed in their respective cells. Thus, the joint probability density function (PDF) of the location of any macrocell mobile user located at $(r, \theta)$ from its serving macrocell $\mathrm{BS}$ can be given as

$$
p(r, \theta)=\frac{r-R_{0}}{\pi\left(R_{1}-R_{0}\right)^{2}}, \quad p_{\theta}(\theta)=\frac{1}{2 \pi}
$$

where $R_{0} \leq r \leq R_{1}$ and $0 \leq \theta \leq 2 \pi$. Similarly, the joint PDF of the location of any femtocell mobile user which is located at $(\tilde{r}, \tilde{\theta})$ from its serving femto $\mathrm{BS}$ can be given by

$$
p(\tilde{r}, \tilde{\theta})=\frac{\tilde{r}}{\pi R_{n}^{2}}, \quad p_{\tilde{\theta}}(\tilde{\theta})=\frac{1}{2 \pi}
$$

where $0 \leq \tilde{r} \leq R_{n}$ and $0 \leq \tilde{\theta} \leq 2 \pi$ (see Fig. 1 for geometrical representation of distances $R_{2}$ and $R_{1}$ ).

\section{ASE AND AGE OF THE ENERGy AwARE HETEROGENEOUS NETWORK}

In this section, we present the mathematical analysis to compute the ASE and AGE of the energy aware FOE configuration. In addition we derive analytical expressions to compute the bounds on the ASE of the FOE configuration.

\section{A. Area Spectral Efficiency of FOE Configuration}

The ASE is typically defined as the sum of the maximum achievable rates per unit bandwidth per unit macrocell area [13]. We express ASE with reference to the macrocell as the allocated frequencies are reused in the macrocells which are located at a distance $D^{\prime}[\mathrm{m}]$. Therefore, we calculate it over the portion of the area covered by macrocell given as $\pi\left(R_{m}+\right.$ $\left.R_{n}\right)^{2}$. The ASE can then be expressed as follows

$$
\eta=\frac{C_{h}}{\pi w_{h}\left(D^{\prime} / 2\right)^{2}}=\frac{4 C_{h}}{\pi w_{h} R_{u}^{2}\left(R_{m}+R_{n}\right)^{2}}
$$

where $R_{u}$ is the normalized reused factor and $R_{u}=2$ represents a fully loaded cellular network. $C_{h}$ denotes the capacity of heterogeneous network and can be written as

$$
C_{h}=C_{m}+C_{n}=\sum_{l=1}^{L} C_{l_{m}}+\sum_{n=1}^{N} \sum_{f=1}^{F} C_{f_{n}}
$$

where $C_{m}$ and $C_{n}$ [bits/sec] are the mean achievable capacity of $m^{\text {th }}$ macrocell and $N$ femtocells, respectively and $C_{l_{m}}=$ $w_{l_{m}} \log _{2}\left(1+\gamma_{l_{m}}\right)$ is the Shannon capacity of $l^{\text {th }}$ mobile user in the $m^{\text {th }}$ macrocell whereas $C_{f_{n}}=w_{f_{n}} \log _{2}\left(1+\gamma_{f_{n}}\right)$ is the Shannon capacity of $f^{\text {th }}$ mobile user in the $n^{\text {th }}$ femtocell. Since we consider an interference limited system where the thermal noise power is negligible relative to the co-channel interference power [13], the received SIR of the $l^{\text {th }}$ macrocell mobile user in cell $m$, i.e., $\gamma_{l_{m}}$ can be given as

$$
\gamma_{l_{m}}=\frac{P_{l_{m}, B_{m}}^{\mathrm{rx}}(r)}{\sum_{i=1, i \neq m}^{M} P_{l_{i}, B_{m}}^{\mathrm{rx}}(r)}
$$

where $P_{l_{m}, B_{m}}^{\mathrm{rx}}(r)$ is the received power level at $B_{m}$ from the $l^{\text {th }}$ mobile user in cell $m$ and can be written explicitly by substituting (3) in (13) as

$P_{l_{m}, B_{m}}^{\mathrm{rx}}(r)= \begin{cases}P_{\max } K r_{l_{m}, B_{m}}^{-\alpha}\left(1+\frac{r_{l_{m}, B_{m}}}{g}\right)^{-\beta} & r_{l_{m}, B_{m}}>R_{t} \\ P_{0} & r_{l_{m}, B_{m}}<R_{t}\end{cases}$

and $P_{l_{i}, B_{m}}^{\mathrm{rx}}(r)$ is the interfering power level received at $B_{m}$ from the $l^{\text {th }}$ mobile user in the $i^{\text {th }}$ interfering macrocell. Similarly, the uplink SIR of the $f^{\text {th }}$ mobile user in the $n^{\text {th }}$ femtocell can be expressed as follows

$$
\gamma_{f_{n}}=\frac{P_{f_{n}, B_{n}}^{\mathrm{rx}}(\tilde{r})}{\sum_{j=1, j \neq n}^{N} P_{f_{j}, B_{n}}^{\mathrm{rx}}(\tilde{r})}
$$

where $P_{f_{n}, B_{n}}^{\mathrm{rx}}(\tilde{r})$ is the received power at $B_{n}$ from the $f^{\text {th }}$ mobile user located in cell $n$ given as

$P_{f_{n}, B_{n}}^{\mathrm{rx}}(\tilde{r})= \begin{cases}P_{\max } K \tilde{r}_{f_{n}, B_{n}}^{-\alpha}\left(1+\frac{\tilde{r}_{f_{n}, B_{n}}}{g}\right)^{-\beta} & \tilde{r}_{f_{n}, B_{n}}>R_{t} \\ P_{0} & \tilde{r}_{f_{n}, B_{n}}<R_{t}\end{cases}$

and $P_{f_{j}, B_{n}}^{\mathrm{rx}}(\tilde{r})$ is the interfering power level received from the $f^{\text {th }}$ mobile user in the $j^{\text {th }}$ interfering femtocell.

\section{B. Bounds on the ASE of Energy Aware FOE Configuration}

We will now focus on deriving the analytical bounds on the ASE of two tier energy aware FOE configuration. It is important to note that determining the statistics of cumulative interference for two slope path-loss model is computationally intensive due to the arbitrary location of the interferers in the uplink. Therefore, we simplified our analysis by fixing the distance of the interferers and their transmit powers which helps in evaluating the bounds for the worst case and best case interference scenarios. The developed bounds provide useful insights in determining the worst case and best case SIR of heterogeneous networks mathematically.

1) Upper Bound: In order to evaluate the upper bound, we consider macrocell best interference configuration which mandates all cochannel interferers to be located on the far boundary of their respective cells, i.e., at a distance of $D^{\prime}+R_{1}[\mathrm{~m}]$ from the desired mobiles BS while transmitting with a power of $P_{m}=\min \left(P_{\max }, P_{0} K R_{1}^{\alpha}\left(1+\frac{R_{1}}{g}\right)^{\beta}\right)$. On the other 


$$
\begin{aligned}
& \bar{\gamma}_{l, m}^{ \pm}=\left(\frac{A_{m}^{ \pm} P_{0}}{P_{\max } K}-A_{m}^{ \pm} r_{l_{m}, B_{m}}^{-\alpha}\left(1+\frac{r_{l_{m}, B_{m}}}{g}\right)^{-\beta}\right) \mathbb{U}\left(R_{t}-r_{l_{m}, B_{m}}\right)+A_{m}^{ \pm} r_{l_{m}, B_{m}}^{-\alpha}\left(1+\frac{r_{l_{m}, B_{m}}}{g}\right)^{-\beta} \\
& \bar{\gamma}_{f, n}^{ \pm}=\left(\frac{P_{0}}{g^{\beta} P_{n} K S^{ \pm}}-\frac{P_{\max } \tilde{r}_{f_{n}, B_{n}}^{-\alpha}\left(1+\frac{\tilde{r}_{f_{n}, B_{n}}}{g}\right)^{-\beta}}{g^{\beta} P_{n} K S^{ \pm}}\right) \mathbb{U}\left(R_{t}-\tilde{r}_{f_{n}, B_{n}}\right)+\frac{P_{\max } \tilde{r}_{f_{n}, B_{n}}^{-\alpha}\left(1+\frac{\tilde{r}_{f_{n}, B_{n}}}{g}\right)^{-\beta}}{g^{\beta} P_{n} K S^{ \pm}}
\end{aligned}
$$

where $A_{m}^{ \pm}=\frac{P_{\max }\left(D^{\prime} \pm R_{1}\right)^{\alpha}\left(g+D^{\prime} \pm R_{1}\right)^{\beta}}{g^{\beta} P_{m}(M-1)}$, and $\mathbb{U}($.$) is the unit step function.$

hand, the femtocell interferers are considered to be located at the edge of their respective cells as illustrated in Fig. 1, while transmitting with the power $P_{n}=\min \left(P_{\max }, P_{0} K R_{n}^{\alpha}\left(1+\frac{R_{n}}{g}\right)^{\beta}\right)$. However, determining the exact best edge with respect to the BS of interest is not simple in femtocell setup, therefore for sake of simplicity we categorized the femtocell interferers roughly into two groups based on their distances from the BS of interest (See Fig. 1).

2) Lower Bound: In order to evaluate the lower bound, we consider macrocell worst interference configuration which mandates all cochannel interferers to be located on the near boundary of their respective cells, i.e., at a distance of $D^{\prime}-R_{1}[\mathrm{~m}]$ from the desired mobiles BS while transmitting with $P_{m}$. Whereas the femtocell interferers are considered to be located at the edge of their respective cells as illustrated in Fig. 1, while transmitting with $P_{n}$.

In order to determine the worst/best edge with respect to the femto BS of interest we will now explain the details of the two interferer group. The first group (Group 1) corresponds to the group of interferers which are relatively located near the femto BS of interest (See Fig. 1), therefore by applying the cosine law, the worst and best case distance $d_{1, n}^{ \pm}$for Group 1 femtocell interferers can be given as

$$
d_{1, n}^{ \pm}=R \sqrt{2} \sqrt{1-\cos \left(\frac{2 \pi n}{N} \pm \frac{\pi}{N}\right)}
$$

where $n$ denotes the integer number by which femto interferer BS is angularly spaced from the femto BS of interest, $1 \leq$ $n \leq \frac{N}{4} \quad \forall N \in$ Even and $1 \leq n \leq \frac{N-1}{4} \quad \forall N \in$ Odd. The second group (Group 2) corresponds to the group of interferers which are relatively located far from the femto BS of interest, therefore $d_{2, n}^{ \pm}$for Group 2 femtocell interferers is given as

$$
d_{2, n}^{ \pm}=\sqrt{R_{m}^{2}+\left(R_{m} \pm R_{n}\right)^{2}-2 R_{m}\left(R_{m} \pm R_{n}\right) \cos \frac{n 2 \pi}{N}}
$$

where $\frac{N}{4}+1 \leq n \leq \frac{N}{2}-1 \forall N \in$ Even and $\frac{N-1}{4}+1 \leq$ $n \leq \frac{N-1}{2}-1 \forall N \in$ Even. By using the symmetry of the FOE configuration, the distances of the remaining half interferers can be computed using (17) and (18). Conditioned on the location of the desired mobile user in macrocell, the worst and best case $\operatorname{SIR}\left(\bar{\gamma}_{l, m}^{ \pm}\right)$can then be derived as:

$$
\bar{\gamma}_{l, m}^{ \pm}= \begin{cases}\frac{\left(D^{\prime} \pm R_{1}\right)^{\alpha}\left(g+D^{\prime} \pm R_{1}\right)^{\beta} P_{0}}{K(M-1) P_{m} g^{\beta}} & r_{l_{m}, B_{m}} \leq R_{t} \\ \frac{P_{\max }\left(D^{\prime} \pm R_{1}\right)^{\alpha}\left(g+D^{\prime} \pm R_{1}\right)^{\beta}}{r_{l_{m}, B_{m}}^{\alpha} g^{\beta}\left(1+\frac{r_{l_{m}, B_{m}}}{g}\right)^{\beta}(M-1) P_{m}} & r_{l_{m}, B_{m}}>R_{t}\end{cases}
$$

Similarly, the worst case and best case SIR which is conditioned on the location of the desired mobile user in femtocell is given as follows:

$$
\bar{\gamma}_{f, n}^{ \pm}= \begin{cases}\frac{P_{0}}{g^{\beta} P_{n} K S^{ \pm}} & \tilde{r}_{f_{n}, B_{n}} \leq R_{t} \\ \frac{P_{\max } \tilde{r}_{f_{n}, B_{n}}^{-\alpha}\left(1+\frac{\tilde{r}_{f_{n}, B_{n}}}{g}\right)^{-\beta}}{g^{\beta} P_{n} K S^{ \pm}} & \tilde{r}_{f_{n}, B_{n}}>R_{t}\end{cases}
$$

where

$$
S^{ \pm}=\sum_{n=1}^{N / 4} \frac{2\left(d_{1, n}^{ \pm}\right)^{-\alpha}}{\left(g+d_{1, n}^{ \pm}\right)^{\beta}}+\sum_{n=1+N / 4}^{N / 2-1} \frac{2\left(d_{2, n}^{ \pm}\right)^{-\alpha}}{\left(g+d_{2, n}^{ \pm}\right)^{-\beta}}
$$

The SIR of desired macro and femto cell mobile user in (19) and (20) can be written in more compact form at the top of this page. Finally, averaging over the distribution of the location of the desired mobile user in macrocell and femtocell, the worst case and best case Shannon capacity can be given as

$$
\begin{gathered}
C_{l_{m}}^{ \pm}=\int_{0}^{R_{m}-R_{n}} \log _{2}\left(1+\bar{\gamma}_{l_{m}}^{ \pm}\right) p_{r}(r) d r \\
C_{f_{n}}^{ \pm}=\int_{0}^{R_{n}} \log _{2}\left(1+\bar{\gamma}_{f_{n}}^{ \pm}\right) p_{\tilde{r}}(\tilde{r}) d \tilde{r}
\end{gathered}
$$

The integration can be solved using mathematical software packages such as MATHEMATICA. Finally, the bounds on $\eta$ of the FOE configuration can be given using (12) as

$$
\eta^{ \pm}=\frac{4\left(w_{m} C_{l, m}^{ \pm}+N w_{n} C_{f, n}^{ \pm}\right)}{w_{t} \pi R_{u}^{2}\left(R_{m}+R_{n}\right)^{2}}
$$

\section{Exact Area Green Efficiency of FOE Configuration}

The AGE of a two tier heterogeneous network is defined as the aggregate power savings per unit macrocell area while maintaining a certain signal power received at the BS receiver. Mathematically, the AGE of two tier hetergoneous network can be expressed as

$$
\mathrm{AGE}=\frac{\mathcal{P}_{m}+\mathcal{P}_{n}}{\pi\left(R_{m}+R_{n}\right)^{2}}
$$

In order to calibrate the AGE of the energy aware FOE configuration, we guarantee the received signal power of $P_{0}$ at both femtocell and macrocell BSs. The total power savings of $L$ macrocell mobile users $\mathcal{P}_{m}$ can be determined as $\mathcal{P}_{m}=\sum_{l=1}^{L} \mathcal{P}_{l_{m}}$ where $\mathcal{P}_{l_{m}}$ is the power saving of the $l^{\text {th }}$ mobile user located in cell $m$ and can be given as follows

$$
\mathcal{P}_{l_{m}}= \begin{cases}0 & r_{l_{m}, B_{m}}>R_{t} \\ P_{\max }-P_{0} \frac{r_{l_{m}, B_{m}}^{\alpha}\left(1+\frac{r_{l_{m}, B_{m}}}{g}\right)^{\beta}}{K} & r_{l_{m}, B_{m}}<R_{t}\end{cases}
$$


Similarly the total power savings of $N F$ femtocell mobile users $\mathcal{P}_{n}$ can be given as $\mathcal{P}_{n}=\sum_{n=1}^{N} \sum_{f=1}^{F} \mathcal{P}_{f_{n}}$ where $\mathcal{P}_{f_{n}}$ is the power saving of the $f^{\text {th }}$ mobile user located in cell $n$ and can be given as follows

$$
\mathcal{P}_{f_{n}}= \begin{cases}0 & \tilde{r}_{f_{n}, B_{n}}>R_{t} \\ P_{\max }-P_{0} \frac{\tilde{r}_{f_{n}, B_{n}}^{\alpha}\left(1+\frac{\tilde{r}_{f_{n}, B_{n}}}{g}\right)^{\beta}}{K} & \tilde{r}_{f_{n}, B_{n}}<R_{t}\end{cases}
$$

In general, a closed form expression for $\mathcal{P}_{l_{m}}$ given in (26) can be derived as:

$\mathcal{P}_{l_{m}}=P_{\max } R_{t}-\frac{2(-1)^{-\alpha}}{R_{m}^{2}}\left(\frac{P_{0}}{K} g^{2+\alpha} \mathcal{B}\left(-R_{t} / g, 2+\alpha, 1+\beta\right)\right)$

where $\mathcal{B}(\cdot, \cdot, \cdot)$ is the incomplete beta function. Similarly, closed form expression for (27) can be derived as:

$\mathcal{P}_{f_{n}}=P_{\max } R_{t}-\frac{2(-1)^{-\alpha}}{R_{n}^{2}}\left(\frac{P_{0}}{K} g^{2+\alpha} \mathcal{B}\left(-R_{t} / g, 2+\alpha, 1+\beta\right)\right)$

\section{Simulation Results And Discussions}

In this section, we quantify the achievable ASE and AGE gain of the energy aware FOE configuration compared to macro-only and macro-femto UDF deployment through simulations. The simulation parameters are summarized in Table 1. The number of mobile users are considered to be adaptive with the coverage area, i.e., we ensure 0.001 mobile users per $\mathrm{m}^{2}$. For sake of fair comparison, $N$ is taken to be same for UDF and FOE configurations.

Fig. 2 demonstrates the ASE gain of FOE compared to macro-only configuration with and without PC. The ASE gain in FOE configuration is more significant within $R_{t}=422 \mathrm{~m}$ compared to the UDF configuration. This is due to the fact that FOE deployment restricts only the cell-edge mobile users to communicate with the femtocells which enhances the overall network ASE compared to UDF configuration. More precisely, UDF configuration allow femto BSs to be deployed in the cell center also which causes an under-utilization of the macro BS capabilities (existing infrastructure). Thus, the performance degradation due to the cell-edge mobile users still exist in the UDF deployment. Due to the weaker channel gains of the mobile users in the large macrocells, the degradation of ASE with $R_{m}$ is also obvious. However, as $R_{m}$ increases beyond $R_{t}$, the proportion of mobile users transmitting with $P_{\max }$ in macrocell increases which enhances the co-channel interference. Thus, the effectiveness of small femtocell tier, i.e., $R_{n}=50 \mathrm{~m}$ compared to the region $R_{1}-R_{t}$ is reduced.

Fig. 3 demonstrates the significant AGE gains of the FOE configuration compared to the macro-only and UDF deployments. Since the deployment of femtocells at the macrocell edge in FOE configuration mandates a reduction in the number of edge mobile users transmitting with the maximum power, the AGE gains are self-explanatory in FOE configuration relative to UDF configuration. However, this effect is more dominant within the threshold distance $R_{t}=422 \mathrm{~m}$ which is due to the fact that the increase of $R_{m}$ until $R_{t}$ increases the number of energy efficient users in both UDF and FOE deployments, however, the energy efficiency of cell-edge users in FOE is higher than the UDF deployment. Nonetheless, this gain of FOE increases till $R_{t}=422 \mathrm{~m}$ beyond which all macrocell mobile users start transmitting with $P_{\max }$ in UDF and FOE configurations and the probability of UDF deployment in non-green area also increases which is equally beneficial as FOE configuration, therefore the effectiveness of FOE slowly disappears beyond $R_{t}$.

In general, the network AGE is found to degrade with the increase of $R_{m}$ which is due to the increase in the proportion of mobile users beyond $R_{t}$ which are transmitting with their maximum power. Therefore, beyond $R_{t}$ the power savings settle down at a specified level as demonstrated in Fig. 4(a). However, small increase of power savings can still be observed due to the increase in the number of femtocells $N$ at the edge and inturn the number of mobile users at the edge of macrocells. The relative AGE gain in percentage with reference to the macro-only configuration is also computed in Fig. 4(b) to illustrate the effective increase in AGE. Fig. 5 presents the theoretical bounds on the ASE of FOE configuration. These bounds provide insights on the gain in ASE of the desired mobile user suffering from the worst case or the best case interference configurations. We can observe that the upper bound is quite tight in FOE configuration which is the scenario in which the macrocell and femtocell interferer are located at the far edge of the interfering cell while transmitting with the adaptive power. The tighter upper bound can therefore be utilized to approximate the ASE of two tier heterogeneous networks numerically.

\section{CONCLUSION}

In this paper, we critically evaluated the area green efficiency (AGE) and area spectral efficiency (ASE) of a macroonly and two macro-femto network deployments (i) femto-onedge (FOE) (ii) uniformly distributed femtocell (UDF). FOE deployment has been shown to provide significant ASE and AGE gains compared to other deployments especially within the threshold distance $\left(R_{t}\right)$ as the users located within the threshold region are able to maintain a desired signal power received at the BS by fully compensating their path loss which is based on their distance from the serving BS. Extensions to the shadowing and fading environments has been covered in the upcoming journal version.

TABLE I

NUMERICAL VALUES OF SIMULATION PARAMETERS [3]

\begin{tabular}{c|c|c}
\hline Simulation parameter & Femtocell & Macrocell \\
\hline Transmit power $\left(P_{\max }\right)$ & $0.8 \mathrm{~W}$ & $0.8 \mathrm{~W}$ \\
\hline Cell radius $(R)$ & $50 \mathrm{~m}$ & $200-600 \mathrm{~m}$ \\
\hline Path-loss exponent $(\alpha)$ & 1.8 & 2.1 \\
\hline Additional path-loss exponent $(\beta)$ & 1.8 & 2.1 \\
\hline BS antenna height $\left(h_{\mathrm{rx}}\right)$ & $12.5 \mathrm{~m}$ & $25 \mathrm{~m}$ \\
\hline Mobile antenna height $\left(h_{\mathrm{tx}}\right)$ & $2 \mathrm{~m}$ & $2 \mathrm{~m}$ \\
\hline Reference distance $\left(R_{0}\right)$ & - & $100 \mathrm{~m}$ \\
\hline Received Power $\left(P_{0}\right)$ & $0.8 \mu \mathrm{W}$ & $0.8 \mu \mathrm{W}$ \\
\hline Breakpoint distance $(g)$ & $300 \mathrm{~m}$ & $600 \mathrm{~m}$ \\
\hline Threshold distance $\left(R_{t}\right)$ & $667 \mathrm{~m}$ & $422 \mathrm{~m}$ \\
\hline System Bandwidth $\left(w_{t}\right)$ & \multicolumn{2}{|c}{$20 \mathrm{MHz}$} \\
\hline Reuse Factor $\left(R_{u}\right)$ & \multicolumn{2}{|c}{1} \\
\hline Femto population factor $(\mathrm{FPF})$ & \multicolumn{2}{|c}{$0.8 \mathrm{~W}$} \\
\hline Path Loss Constant $(K)$ & \multicolumn{2}{|c}{}
\end{tabular}




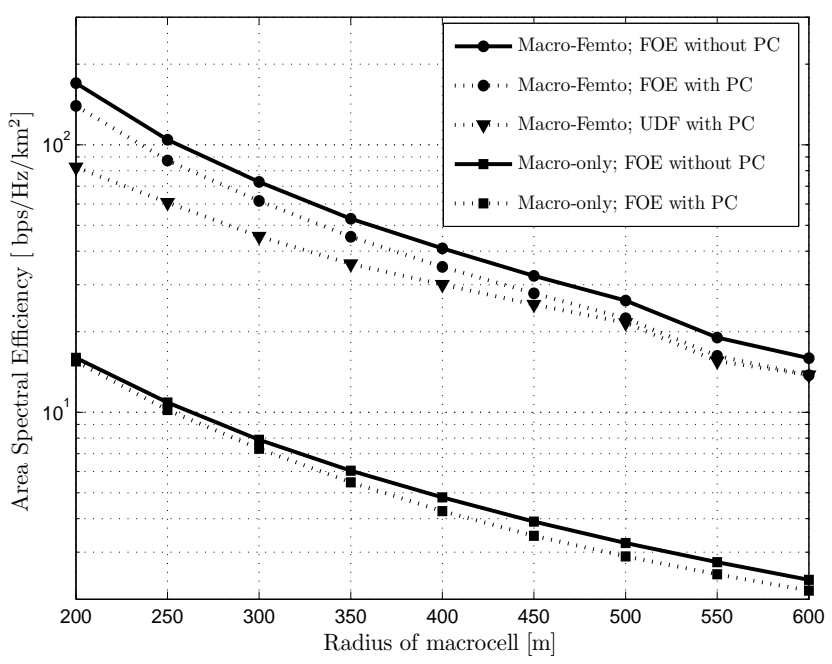

Fig. 2. Comparison of the ASE of macro-only network with two macro-femto configurations (i) Femto-on-edge (FOE) (ii) Uniformly distributed femtocells (UDF) with and without $\mathrm{PC}$ as a function of $R_{m}$.

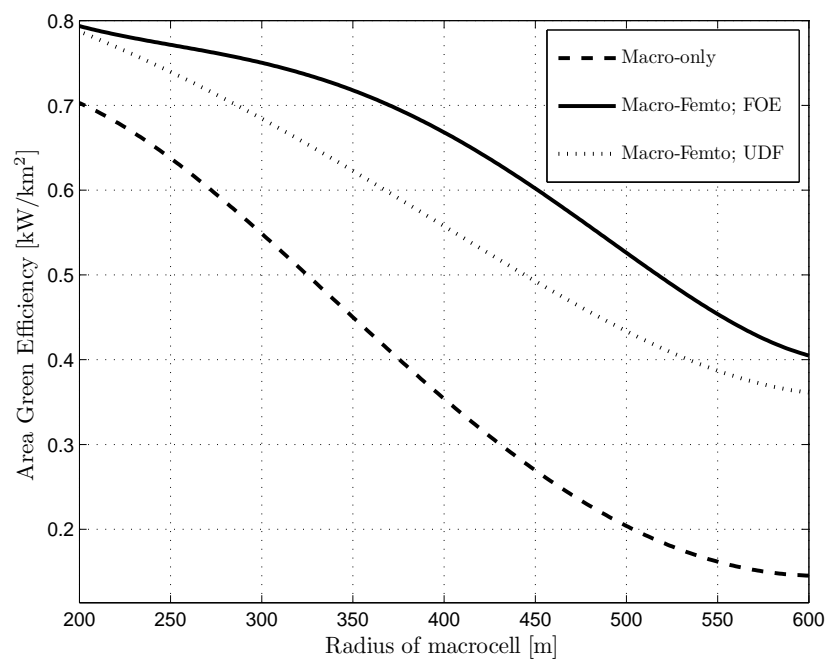

Fig. 3. Comparison of the AGE of macro-only network with two macro-femto configurations (i) Femto-on-edge (FOE) (ii) Uniformly distributed femtocells (UDF) as a function of $R_{m}$.

\section{REFERENCES}

[1] P. Lin, J. Zhang, Y. Chen, and Q. Zhang, "Macro-femto heterogeneous network deployment and management: from business models to technical solutions," IEEE Wireless Communs. Mag., vol. 18, no. 3, pp. 64-70, Jun. 2011.

[2] S. Landstrom, A. Furuskar, K. Johansson, L. Falconetti, and F. Kronestedt, "Heterogeneous networks increasing cellular capacity," Jour. Ericson Review, vol. 89, pp. 4-9, Jan. 2011.

[3] Femto Forum, "An overview of the femtocell concept." [Online]. Available: www.femtoforum.org.

[4] N. Shetty, S. Parekh, and J. Walrand, "Economics of femtocells," in Proc. IEEE Conf. Global Communs., (GLOBECOM'09), pp. 1-5, Honolulu, HI, USA, Dec. 2009

[5] F. Cao, and Z. Fan, "The tradeoff between energy efficiency and system performance of femtocell deployment," in Proc. Intl. Symp. Wireless Commun. Systems, (ISWCS'10), York, UK, Sep. 2010.

[6] V. Chandrasekhar, J. Andrews, and A. Gatherer, "Femtocell networks: a survey," IEEE Communs. Mag., vol. 46, no. 9, pp. 59-67, Sep. 2008.

[7] T. Q. S. Quek, W. C. Cheung, and M. Kountouris, "Energy efficiency analysis of two-tier heterogeneous networks," 11th European Wireless Conference, pp. 1-5, 2011.
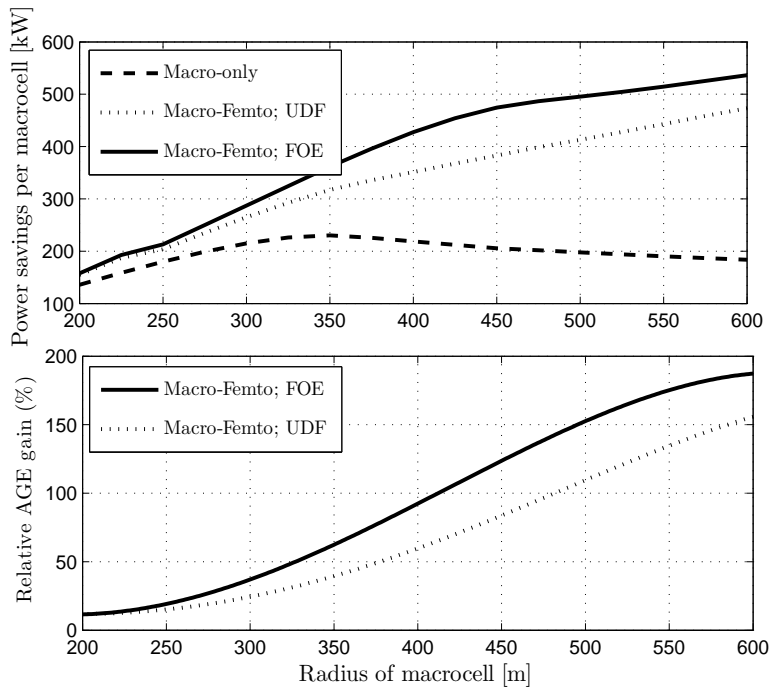

Fig. 4. (a) Total power saved by all mobile users in macro-only network with two macro-femto configurations (i) Femto-on-edge (FOE) (ii) Uniformly distributed femtocells (UDF) (b) Relative percentage gain in the AGE of two macro-femto configurations compared to the macro-only network.

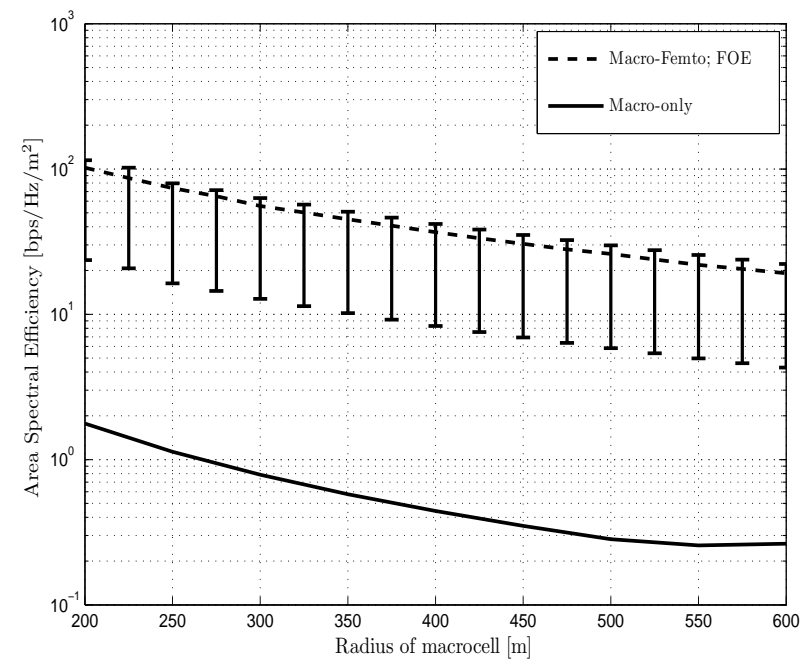

Fig. 5. Analytical bounds on the ASE of FOE configuration as a function of the radius of macrocell.

[8] Vodafone Chair, "Cool cellular-energy efficient network architectures and transmission methods," avaiable at http://www.vodafonechair.com/research/projects cool cellular.html.

[9] K. Lahiri, A. Raghunathan, S. Dey, and D. Panigrahi , "Battery-driven system design: a new frontier in low power design," in Proc. Intl. Conf. on VLSI Design, pp. 261-267, Bangalore, India, Jan. 2002.

[10] G.W. Miao, N. Himayat, Y. Li, and A. Swami, "Cross-layer optimization for energy-efficient wireless communications: a survey," Wiley Wireless Communications and Mobile Computing, vol. 9, no. 4, pp. 529-542, 2009.

[11] M. Z. Shakir and M.-S. Alouini, "On the area spectral efficiency improvement of heterogeneous network by exploiting the integration of macro-femto cellular networks," in Proc. IEEE Intl. Conf. Communs., (ICC'12), pp. 1-6, Ottawa, Canada, Jun. 2012.

[12] A. Simonsson and A. Furuskar, , "Uplink power control in LTE overview and performance, principles and benefits of utilizing rather than compensating for SINR variations," in Proc. IEEE Vehicular Technology Conference, (VTC'08 Fall), Calgary, Alberta, Sept. 2008.

[13] M.-S. Alouini, and A. Goldsmith, "Area spectral efficiency of cellular mobile radio systems," IEEE Trans. Vehicular Technology, vol. 48, no. 4, pp. 1047-1066, Jul. 1999. 